This is the peer reviewed version of the following article:

Fiorenza, L, Benazzi, S, Oxilia, G, Kullmer, O. Functional relationship between dental macrowear and diet in Late Pleistocene and recent modern human populations. Int J Osteoarchaeol. 2018; 28: 153-161, which has been published in final form at https://doi.org/10.1002/oa.2642. This article may be used for non-commercial purposes in accordance with Wiley Terms and Conditions for Use of Self-Archived Versions. This article may not be enhanced, enriched or otherwise transformed into a derivative work, without express permission from Wiley or by statutory rights under applicable legislation. Copyright notices must not be removed, obscured or modified. The article must be linked to Wiley's version of record on Wiley Online Library and any embedding, framing or otherwise making available the article or pages thereof by third parties from platforms, services and websites other than Wiley Online Library must be prohibited.

This item was downloaded from IRIS Università di Bologna (https://cris.unibo.it/)

When citing, please refer to the published version. 


\title{
Functional relationship between dental macrowear and diet in Late Pleistocene and recent modern human populations
}

\author{
Luca Fiorenza $^{1,2}$ | Stefano Benazzi ${ }^{3,4}$ | Gregorio Oxilia ${ }^{3,5}$ | Ottmar Kullmer ${ }^{6,7}$ \\ ${ }^{1}$ Department of Anatomy and Developmental Biology, Monash University, Melbourne, VIC, Australia \\ ${ }^{2}$ Earth Sciences, University of New England, Armidale, NSW, Australia \\ ${ }^{3}$ Department of Cultural Heritage, University of Bologna, Bologna, Italy \\ ${ }^{4}$ Department of Human Evolution, Max Planck Institute for Evolutionary Anthropology, Leipzig, Germany \\ ${ }^{5}$ Department Biology, University of Florence, Florence, Italy \\ ${ }^{6}$ Senckenberg Research Institute, Frankfurt, Germany \\ ${ }^{7}$ Department of Paleobiology and Environment, Institute of Ecology, Evolution, and Diversity, Johann Wolfgang Goethe University, Frankfurt, Germany \\ Correspondence \\ Dr Luca Fiorenza, Department of Anatomy and Developmental Biology, Monash University, Melbourne, VIC 3800 Australia. Email: luca.fiorenza@monash.edu \\ Funding information \\ Faculty of Medicine, Nursing and Health Sciences, Grant/Award Number: SGS16-0344
}

\begin{abstract}
Many aspects of diet and behaviour can be gleaned from dental wear, including environmental conditions, food-fracture properties, food processing techniques, and cultural habits. Specifically, the angulation (flat vs. steep) of molar wear has been used as an indicator of food toughness and has also been implicated in the use of grinding stones and pottery in food processing. In this work, we focus on the sequential phases of the power stroke of mastication in a functional way, measuring the inclination of molar wear facets through the occlusal fingerprint analysis method. Specifically, we have calculated the angulation of wear facets in upper and lower molars of Palaeolithic humans, extant hunter-gatherers, and proto-farmers to discern differences between groups with different diets.

Contrary to previous analyses, our study shows that the molars of Late Pleistocene specimens are characterized by significantly steeper angles than those of modern hunter-gatherers.

The flat molar wear found in the latter group could be related to the excessive mixture of exogenous materials accidentally introduced into their foods, as indicated by ethnographic evidence. On the contrary, the steep wear angles characterizing the Palaeolithic group are probably associated with the consumption of a less abrasive diet, which could be ultimately due to food preparation techniques that incorporated less dust and grit into their diets.
\end{abstract}

KEYWORDS

early farmers, food preparation, hunter-gatherers, Neanderthals, wear facets 


\section{INTRODUCTION}

The study of tooth wear has been an invaluable tool for the reconstruction of dietary and cultural habits in prehistoric populations and extinct human species (e.g., El Zaatari, Grine, Ungar, \& Hublin, 2011; Fiorenza, 2015; Fiorenza, Bennazi, Tausch, et al., 2011; Fiorenza et al., 2015; Fiorenza, Benazzi, \& Kullmer, 2011; Fiorenza \& Kullmer, 2013, 2015; Hinton, 1982; Molnar, 1972; Smith, 1984). Dental wear can be examined at two different scales: macrowear (visible to the naked eye or at low magnification) and microwear (visible only under microscope). Several different factors can contribute to the progres-sion of dental wear, including diet, food processing techniques, and exogenous abrasives, and untangling the influence of each factor can be difficult (Addy \& Shellis, 2006). That said, several archaeological and ethnographic studies have highlighted the importance of specific cultural and environmental factors in the formation of specific wear patterns in historic and modern hunter-gatherer human populations
(Hinton, 1982; Kaidonis, Townsend, \& Richards, 1993; Molnar, 1971, 1972). In particular, it is hypothesized that food preparation practices can introduce a large quantity of abrasive exogenous materials, such as sand, dust, or ash, which may influence the wear patterns of these human populations (Hinton, 1982; Kaidonis et al., 1993; Molnar, 1971, 1972; Watson, 2008). Smith (1984) examined the patterns of molar wear in hunter-gatherers and agriculturalists and associated changes in human subsistence by measuring their wear plane angles. For example, hunter-gatherers and agriculturalists are characterized by flatter and more oblique wear, respectively. This is related with dif-ferent food preparation techniques that reduce food toughness or fibrousness (Smith, 1984). More interestingly, wear plane angles between various hunter-gatherer groups were similar despite regional differences in diet. Similarly, Le Luyer, Rottier, and Bayle (2014) examined enamel thickness distribution and variation in Neolithic and Medieval populations, and these researchers found a correlation between oblique tooth wear pattern and food preparation techniques. 
Thus, it seems that the overall angulation of molar wear can distinguish between human populations that adopted significantly different subsistence strategies (e.g., hunter-gatherers vs. agriculturalists) but is unable to detect smaller scale differences in diet in the hunter-gatherer groups. Additionally, analyses of surface microwear texture complexity among modern hunter-gatherer and Paleolithic human teeth were found to be correlated with the level of abrasiveness with food preparation methods associated with cultural changes in the human diets (El Zaatari, 2008, 2010; El Zaatari \& Hublin, 2014). This leaves open the question of what general wear plane angles can really tell us about changes in diet, culture, and technology.

In our study, we tested whether the angulations of wear facets can discriminate between human populations from different chronological times that relied on contrasting diets and preparation technologies, using the well-established three-dimensional approach known as occlusal fingerprint analysis (OFA, Kullmer et al., 2009). Wear facets are flat and shiny areas with well-defined borders produced mainly by the attritional contacts between upper and lower teeth (e.g., Every, 1972). The surface of the wear facets is covered with minute subpar-allel striations mirroring the orientation of tooth movements (Kay, 1977). Every facet is matched by a corresponding facet on a tooth in the opposing arch, and when dentin is exposed, it remains flat with no "scooping" (Kaidonis, 2008). The OFA method is based on quantita-tive analyses that measure different wear facet variables and links them with dental function, masticatory movements, culture, and diets (Fiorenza, 2015; Fiorenza et al., 2015; Fiorenza, Benazzi, Tausch, et al., 2011; Fiorenza, Benazzi, Tausch, Kullmer, \& Schrenk, 2010; Fiorenza, Benazzi, Viola, et al., 2011; Fiorenza \& Kullmer, 2013, 2015, 2016; Harvati et al., 2013; Kullmer et al., 2009; Oxilia et al., 2015; Ulhaas, Kullmer, \& Schrenk, 2007).

Unlike microwear, dental macrowear is a cumulative process that occurs throughout the individual's lifetime and is not influenced by the "The Last Supper" effect (Grine, 1986; Janis, 1990). One of the major advantages of this approach is the use of high-resolution 3D models of teeth, which allows detailed spatial measurements of the wear facet plane angles, thereby overcoming the limitations of 2D analyses seen in Smith's work (1984). Moreover, our virtual approach takes into account the functional aspects of tooth macrowear during the sequential phases (power stroke) of the masticatory cycle (which was not examined by Smith, 1984), providing more detailed information on the attritional occlusal jaw movements and mecha-nisms behind the formation of each wear facet and thus permitting the differentiation between dietary and nondietary movements and wear (Fiorenza, 2015; Fiorenza, Benazzi, Kullmer, 2011; Fiorenza \& Kullmer, 2013, 2015, 2016). We have shown that relative facet areas (obtained by dividing each facet area by the total occlusal wear area) of hunter-gatherer molars differ between groups that consumed different foods and are therefore a good indicator of diet (Fiorenza, Benazzi, Tausch, et al., 2011). Moreover, we have also demonstrated that the formation and general wear facet pattern in Pleistocene and modern humans are not influenced by tooth morphology (Fiorenza, Benazzi, Viola, et al., 2011), corroborating previous works, which found that differences in dental morphology do not generate differences in occlusal wear patterns if two mammalian taxa consume similar diets (Janis, 1990).
We measure and compare the wear facet angulation of Paleolithic human molars with those of recent modern hunter-gatherers and early farmers (with known diets and known cultural habits). Because this approach is limited to molars with a moderate degree of wear (Fiorenza, 2009), we could only examine individuals at a young age, ranging between young adults (with third molars erupted), adolescent (with second molars erupted), and children (with the first molars erupted and in full occlusion). Preliminary results have shown that at more advanced wear stages, there are no significant differences in wear facet angles between human groups with different diets and different subsistence strategies (Fiorenza, 2009). Primary tooth morphology becomes obliterated as wear increases; this causes wear facets to coalesce and renders their identification impossible. Although this limitation may prevent the use of large sample sizes (in particular for the modern hunter-gatherer groups), it is important to note that increased longevity in humans became common very late in our evolu-tion. For example, Caspari and Lee (2004) estimated that only $33 \%$ of Neanderthal individuals of their sample reached adulthood, whereas $67 \%$ died at a young age. Thus, our approach allows us to take into account a large proportion of Palaeolithic human populations, some-thing that it was not possible, for instance, in Smith's study (1984), where only 14 Palaeolithic specimens were analyzed.

We test the null hypothesis of no correlation between the angula-tion of wear facets and diet (Hypothesis 0 ), and we investigated whether functional wear facet angles can be employed for larger scale subsistence divisions (e.g., hunter-gatherer vs. farming) that are related to major differences in food preparation methods and technology (Hypothesis 1). If the null hypothesis is rejected, hunter-gatherer groups that rely on different diets should be also characterized by different wear facet angles. If this hypothesis is instead accepted, we can examine other possible causes that are beyond the determination of flat versus oblique wear facets. This information can help us to bet-ter understand the mechanisms and processes behind the formation of tooth wear in human populations and to evaluate whether occlusal macrowear patterns contain information on culturally related changes in the human diet.

\section{MATERIAL AND METHODS}

The sample used in this study consists of maxillary and mandibular molars of prehistoric humans, modern hunter-gatherers, and protofarmers. The specimens listed in Table 1 include fossil individuals from the Middle and Upper Palaeolithic (PALAEO, $n=34$ ), recent huntergatherers (MHG, $n=15$ ), and Natufians (FARM, $n=7$ ) from the Levant who lived during the transition from hunting and gathering to early farming (Bar-Yosef, 2011; Bowles \& Choi, 2013; Table 1). The recent sample consists of populations with known diets, including predominantly meat-eating groups such as Inuit $(n=4)$ and Vancouver islanders $(n=5)$, and also mixed-diet hunter-gatherers such as the Khoe-San $(n=$ $6)$. The Inuit diet is almost entirely composed of animal proteins, including fish, sea mammals, terrestrial herbivores, birds, and, occasionally, vegetable materials such as berries (Baarrengaard, 1949; Gotfredsen, 1997; Pedersen, 1947; Pedersen \& Scott, 1951). These populations often eat their food raw but sometimes frozen or dried 
TABLE 1 List of Palaeolithic humans, modern hunter-gatherers, and proto-farmers used

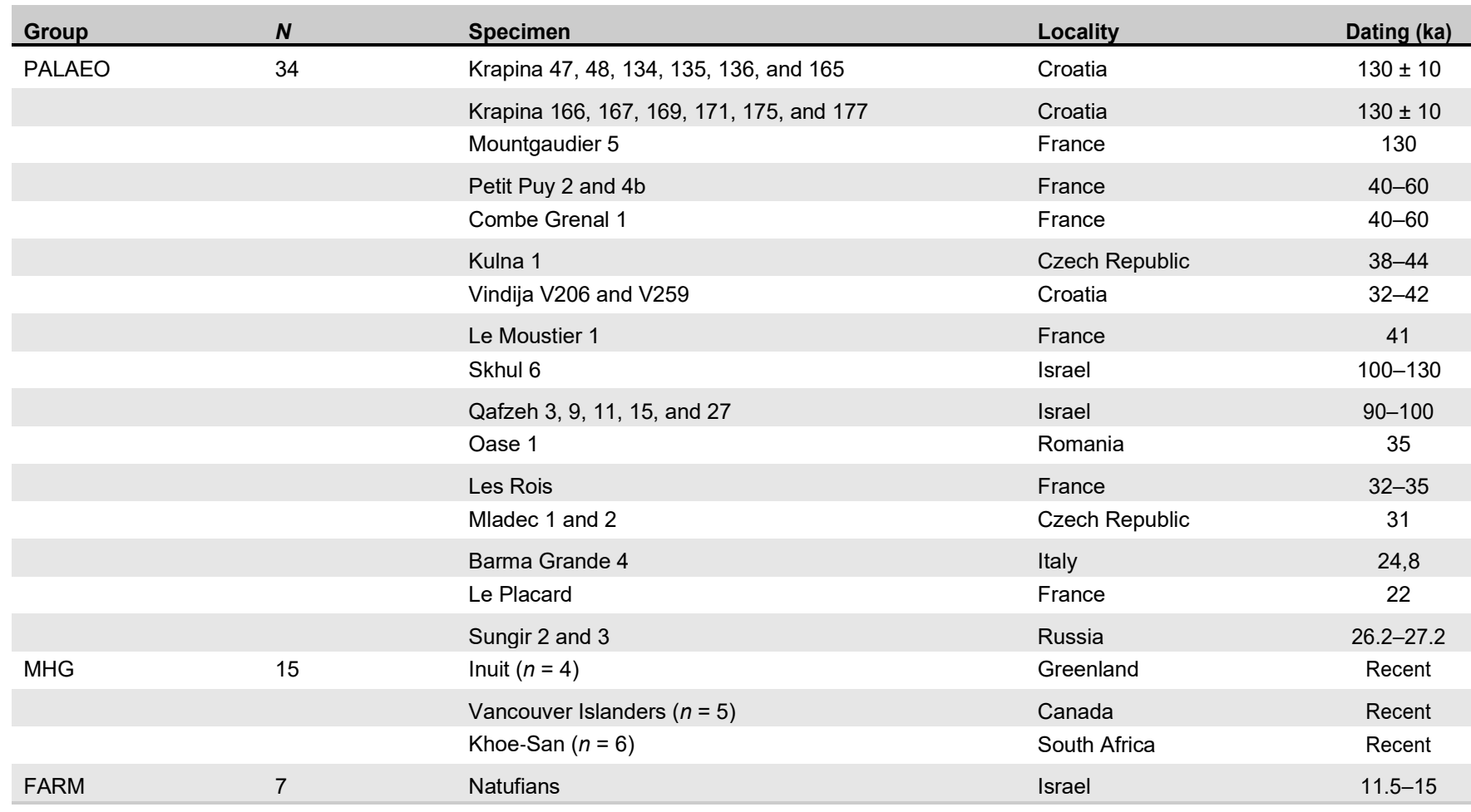

Note. Groups: Palaeolithic individuals (PALAEO), modern hunter-gatherers (MHG), and proto-farmers (FARM). Numbers of MHG individuals are shown in parentheses.

(Baarrengaard, 1949; Gotfredsen, 1997; Pedersen, 1947; Pedersen \& Scott, 1951). The subsistence economy of Vancouver islanders was mainly based on the exploitation of various animal proteins but dominated by various species of salmon, which were abundant and available at predictable times and places (Ames \& Maschner, 1999). They were consumed fresh with the remainder dried, smoked, and stored. Finally, the Khoe-San people were mostly gatherers rather than hunters; their diets consisted predominantly of plant foods $(60$ $80 \%$ ), whereas meat was only of secondary dietary importance because animals were scarce and their presence unpredictable (Lee, 1973, 1978; Lee \& DeVore, 1976; O'Dea et al., 1991; Sahlins, 1968; Silberbauer, 1981).

\subsection{Identification of wear facets}

The identification of occlusal wear facets is based on the terminology proposed by Maier and Schneck (1981), who recognized 13 complementary pairs of facets in hominoid molars (Kullmer et al., 2009). We divided the facets into three groups based on their contri-bution to the power stroke phases of the chewing cycle: Buccal Phase I facets (coloured in blue; Facets 1, 2, 3, and 4), Lingual Phase I facets (coloured in red; Facets 5, 6, 7, and 8), and Phase II facets (coloured in green; Facets 9, 10, 11, 12, and 13; Fiorenza, Benazzi, Tausch, et al., 2011; Janis, 1990; Kay \& Hiiemae, 1974). All mammals chew on one side of the jaw at a time and move the lower jaw in an orbital circuit (Janis, 1990). This orbital circuit consists of two distinct functional steps: puncture-crushing, where the food is initially pulped and tooth-to-tooth contact rarely occurs, and a rhythmic chewing phase (also called power stroke) where crushing and grinding of the food takes place (Kay \& Hiiemae, 1974). During the first part of the power stroke (Phase I), the opposing molar crests tend to slide past each other, and the trapped food is exposed to a shearing action generated by forces parallel to the contact plane. At the end of Phase I, food is compressed (crushing) between basins and cusps of molars (the occlusal force is perpendicular to the contact plane) that are moving in centric occlusion (or maximum intercuspation). Phase II is usually an anterior-medial movement, where the lower molars move out of centric occlusion and the food is processed by grinding (which is the resulting action of the combination of perpendicular and parallel forces to the contact plane). It is during the chewing cycle, after the food is sufficiently softened and reduced in volume, that the surfaces of the upper and lower teeth get into close occlusal contacts and wear facets start to develop.

We further divided the Phase I facets into buccal and lingual proportions following the functional interpretations of Janis (1990) who analyzed the occlusal macrowear patterns in different mammalian groups. The distinction of wear facets into power stroke phases, and the proportions of shearing, crushing, and grinding wear, is particularly important to understand the functional relationship between dental wear and the physical properties of the foods consumed. This can be useful for dietary interpretations as well (Fiorenza, Benazzi, Tausch, et al., 2011). In addition, flat worn areas resulting from tip crushing were also visible on the surface of the major cusp tips (Janis, 1990). However, tip crushing facets were not considered here because their formation is still debated and probably cannot only be attributed to the normal power stroke of the chewing cycle. 
Complementary occlusal wear facets tend to coalesce in advanced wear stages, often preventing their clear identification and contouring. Therefore, we selected only molars with a slight degree of wear based on the amount of cusp removal and dentine exposure (Wear Stage 2; Smith, 1984). However, because most of our modern dental sample is characterized by heavily worn teeth, we also included lower molars in an effort to increase the sample size. We initially tested whether using lower and upper molars affects the obtained result. We also compared the inclinations of first, second, and third molars to investi-gate whether there was any difference by dental element. To accom-plish this, we used upper and lower molars from the same sample (Neanderthals from Krapina, Croatia). We also compared the inclina-tion of upper and lower molars in the modern hunter-gatherer sample (see Supporting Information).

\subsection{Occlusal fingerprint analysis}

Three-dimensional computer models were produced by digitizing high- quality dental casts (Fiorenza, Benazzi, \& Kullmer, 2009) through a white-light scanning system with an $x-y$ resolution of 45 $\mu \mathrm{m}$ (smartSCAN 3D C-5, Breuckmann $\mathrm{GmbH}$ ). Postprocessing of the polygonal models, together with the identification and analysis of wear facets, was carried out using Polyworks ${ }^{\circledR}$ V12 (InnovMetric software), a 3D metrology software. The OFA method can be summarized as encompassing three sequential steps: use of a reference plane, facets identification, and calculation of wear facet plane inclination (Figure 1).

The computation of a reference plane (Step 1) is based on the use of a reference Cartesian $x y$-plane created along the cervical line of each individual tooth by means of the least square best-fit method (Fiorenza et al., 2010; Kullmer et al., 2009; Ulhaas et al., 2007). Wear facets were manually identified and segmented (Step 2) on the surface of the 3D models using the polyline tool of Polyworks $®$ V12 (InnovMetric software). In order to create the plane of each facet, we selected all triangles contained in the facet perimeter and used the best-fit plane function; the inclination (Step 3) is the angle measured between the facet and the reference plane (Kullmer et al., 2009).
Finally, for each specimen, we calculated the average angle of the inclination of every phase of the power stroke.

\subsection{Itatistical analyses}

We calculated the median, standard deviation, and interquartile range of three variables (angles of Buccal Phase I, Lingual Phase I, and Phase

II wear facets) for each sample. Because the sample examined in this study consists of few individuals, which prevent the assumption of a normal distribution, we employed the multivariate and nonparametric one-way permutational multivariate analysis of variance test, which is a based on any distance measure between two or more groups. Statis-tical significance is computed with permutation test of group member-ship ( $n$ $=9,999)$. Finally, for the comparison of Facet 9 inclinations between upper and lower molars, we used a Mann-Whitney univariate test. The statistical analysis was accomplished with the software PAST v.3.13 (PAlaeontological STatistics Hammer, Harper, \& Ryan, 2001).

\section{RESULTS}

\subsection{Analysis of the power stroke}

Overall, Lingual Phase I facets show the greatest variation and steeper inclination values than those of Buccal Phase I and Phase II areas (Figure 2). This is further confirmed by the Mann-Whitney univariate test where a significant difference $(p<.05)$ is found between Buccal and Lingual Phase I facets and between Phase II and Lingual Phase I facets (Table 2). When the wear patterns of the different groups are considered, Palaeolithic humans are characterized by steep Buccal Phase I and Lingual Phase I wear facets and distinctively flat Phase II wear facets (Table 3). On the other hand, the MHG sample displays the lowest wear plane angles in both power stroke phases, whereas the Natufians have equivalent Buccal Phase I and Phase II wear facet inclinations, coupled with steep Lingual Phase I wear angles. The wear plane angles of each group have been illustrated schematically using the transverse occlusal profile (Figure 3), where the power stroke phases are depicted by slopes that represent the average wear plane angles given in Table 3.

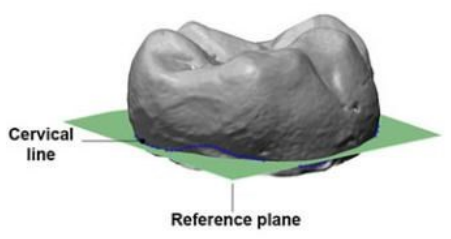

Step 1

Reference plane

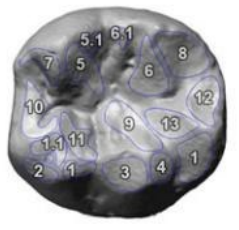

Step 2 Identification

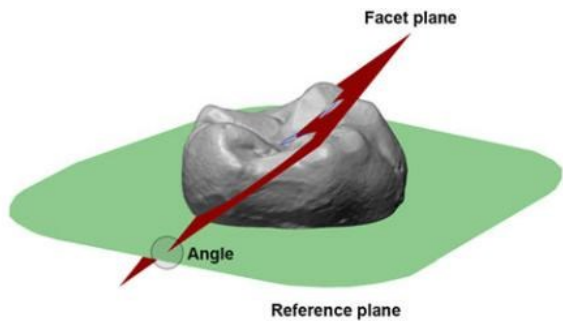

Step 3 Inclination

FIGURE 1 Occlusal fingerprint analysis method: Computation of a reference plane along the cervical line (Step 1), wear facet identification (Step 2) based on Maier and Schneck (1981) labelling system, and inclination, measured between the reference and facet plane (Step 3). Buccal Phase I wear facets (Facets 1, 1.1, 2, 2.1, 3, and 4); Lingual Phase I wear facets (Facets 5, 5.1, 6, 6.1, 7, and 8); Phase II wear facets (Facets 9, 10, 11, 12, and 13) [Colour figure can be viewed at wileyonlinelibrary.com] 


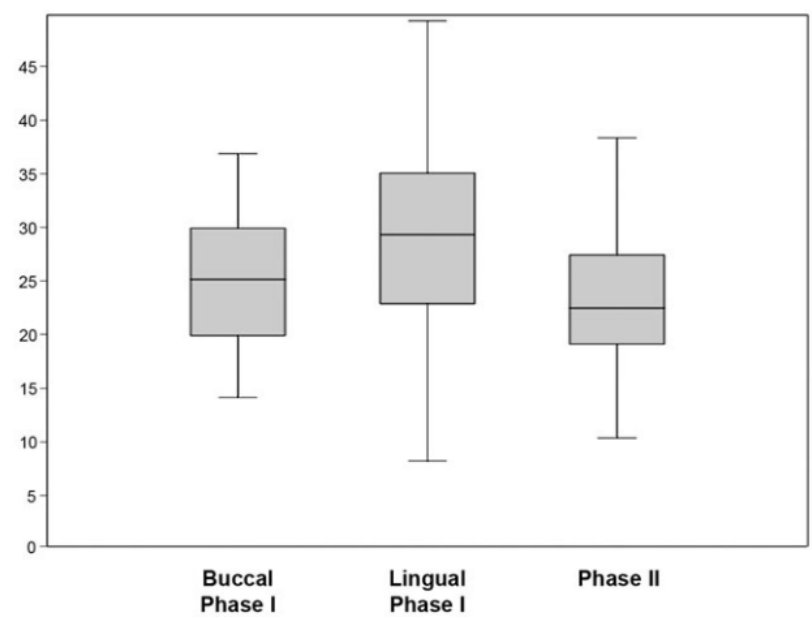

FIGURE 2 Boxplots illustrating the variation in the degree of wear facet inclination within the different chewing phases in the entire sample analyzed in this study $(N=56)$. Buccal Phase I wear facets (Facets $1-4)$, Lingual Phase I wear facets (Facets 5-8), and Phase II wear facets (Facets 9-13)

TABLE 2 Between-group comparisons of wear facet inclinations grouped according to chewing cycle phases (Kay \& Hiiemae, 1974)

\begin{tabular}{llll} 
Facets & Buccal Phase I & Lingual Phase I & Phase II \\
\hline Buccal Phase I & $* * *$ & & \\
Lingual Phase I & $\mathbf{0 . 0 1 1}$ & $* * *$ & \\
Phase II & 0.1989 & $\mathbf{0 . 0 0 0 3}$ & $* * *$
\end{tabular}

Note. Buccal Phase I facets (Facets 1-4), Lingual Phase I facets (Facets 5-8), and Phase II facets (Facets 9-13).

Mann-Whitney univariate test.

Significant $p$ values (<.05) are highlighted in bold.

\subsection{Wear facet inclination and diet}

For testing the first null hypothesis, that there is no correlation between the inclination of wear facets and diet, we analyzed each MHG group individually (Table 4). Inuit molars are marked by extremely flat Buccal Phase I and Phase II facets, with relatively steep Lingual Phase I facets. In contrast, Vancouver Islanders and Khoe-San are characterized by similar values in both chewing phase facets, with the former possessing the flattest wear within the entire MHG sample analyzed in this study. A pairwise comparison between these three MHG groups did not show any statistical significance (Table 5).

As anticipated, if we compare the wear facet inclinations between modern hunter-gatherers and early farmers, we find a statistical significant difference between the two groups $(p=.0314)$, with the former sample characterized by a flatter occlusal wear. However, contrary to
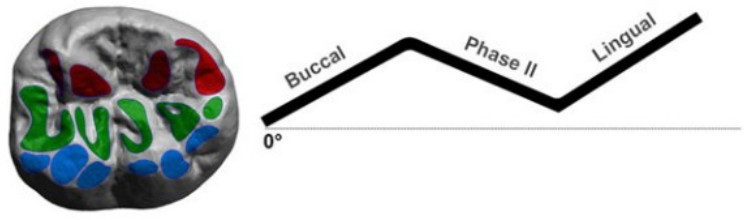

논
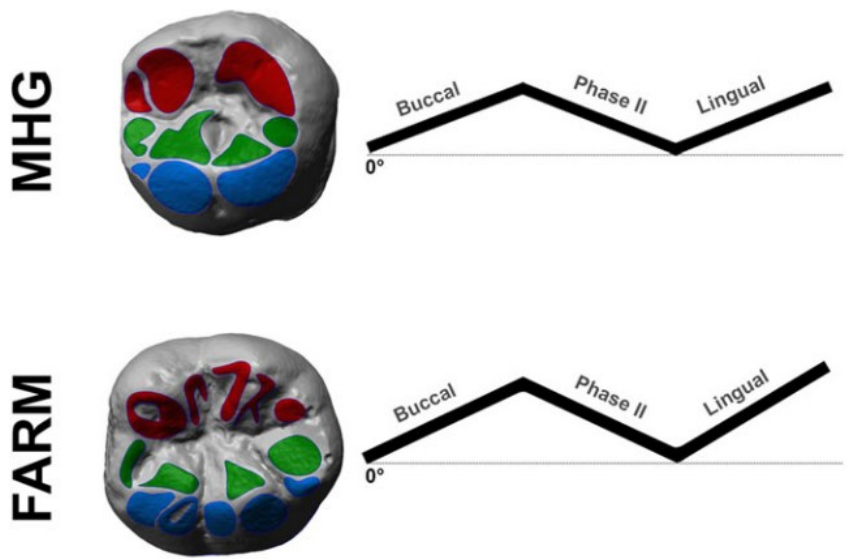

FIGURE 3 Occlusal profile. Digital 3D polygonal models of lower molars in occlusal view showing the different wear facets grouped by chewing cycle phases (Kay \& Hiiemae, 1974): Buccal Phase I wear facets (Facets 1-4; blue), Phase II wear facets (Facets 9-13; green), and Lingual Phase I wear facets (Facets 5-8; red). Occlusal profiles of each polygonal model is shown on the right; the slope (inclination) of the different chewing cycle phases has been calculated using mean angle values (see Table 2). Groups: Palaeolithic individuals (PALAEO), modern hunter-gatherers (MHG), and Natufians (FARM). This figure is available in colour online at wileyonlinelibrary.com/journal/oa [Colour figure can be viewed at wileyonlinelibrary.com]

previous studies, the Palaeolithic group shows a significant more oblique wear than those of MHG individuals $(p=0.0002)$, which it is comparable with those of early farmers (Table 6).

\section{DISCUSSION}

\subsection{Dental macrowear in the Palaeolithic humans}

It has been generally accepted that Palaeolithic humans had a much tougher and more fibrous diet than their modern counterparts (Molnar, 1971, 1972). In particular, Smith (1984) found that Palaeolithic humans tended to show flatter wear than modern hunter-gatherers, displaying the lowest plane angles of the entire sample analyzed in the study. This has suggested that the dentition of Palaeolithic humans underwent the heaviest demand on the masticatory apparatus. However, our study

TABLE 3 Explorative statistical analysis of wear facet inclinations with median, standard deviation (SD), and interquartile range (IQR)

\begin{tabular}{lcccccccrrr} 
& \multicolumn{4}{c}{} & \multicolumn{4}{c}{ Buccal Phase I } & \multicolumn{4}{c}{ Lingual Phase I } & \multicolumn{2}{c}{ Phase II } \\
Group & $\boldsymbol{N}$ & Median & SD & IQR & Median & SD & IQR & Median & SD & IQR \\
\hline PALAEO & 34 & 27.1 & 5.6 & 8.8 & 31.4 & 6.9 & 11.1 & 22.5 & 7.3 & 9.6 \\
MHG & 15 & 20.1 & 5.7 & 7.5 & 21.1 & 7.4 & 15.0 & 22.3 & 4.2 & 5.9 \\
FARM & 7 & 26.5 & 6.6 & 9.3 & 31.2 & 10.8 & 14.0 & 26.7 & 8.4 & 14.2 \\
\hline
\end{tabular}

Note. Group labels follow Table 1. 
TABLE 4 Explorative statistical analysis of wear facet inclinations with median, standard deviation (SD), and interquartile range (IQR) within the modern hunter-gatherers (MHG) group

\begin{tabular}{|c|c|c|c|c|c|c|c|c|c|c|}
\hline \multirow[b]{2}{*}{ Groups } & \multirow[b]{2}{*}{$N$} & \multicolumn{3}{|c|}{ Buccal Phase I } & \multicolumn{3}{|c|}{ Lingual Phase I } & \multicolumn{3}{|c|}{ Phase II } \\
\hline & & Median & SD & IQR & Median & SD & IQR & Median & SD & IQR \\
\hline INUIT & 4 & 21.2 & 3.3 & 6.0 & 21.9 & 5.3 & 8.7 & 21.9 & 1.5 & 2.4 \\
\hline $\mathrm{KHOE}$ & 6 & 19.8 & 3.2 & 5.2 & 22.1 & 5.3 & 8.2 & 24.6 & 5.6 & 6.8 \\
\hline
\end{tabular}

Note. MHG Groups: INUIT (Inuit), VANCOUV. (Vancouver Islanders), and KHOE (Khoe-San).

TABLE 5 Multivariate statistical analysis of wear facet inclinations in the modern hunter-gatherers (MHG) sample

\begin{tabular}{llll} 
Groups & INUIT & VANCOUV & KHOE \\
\hline INUIT & $* * *$ & & \\
VANCOUV & 0.3946 & $* * *$ & \\
KHOE & 0.8329 & 0.5773 & $* * *$ \\
\hline
\end{tabular}

Note. One-way permutational multivariate analysis of variance test Permutation $N=9,999, P$ (same) $=.7173$.

Significantly $p$ values (<.05) are highlighted in bold.

Group labels follow Table 4

TABLE 6 Multivariate statistical analysis of wear facet inclinations within the entire sample analyzed in this study

\begin{tabular}{llll} 
Groups & PALAEO & MHG & FARM \\
\hline PALAEO & $* * *$ & & \\
MHG & $\mathbf{0 . 0 0 0 2}$ & $* * *$ & \\
FARM & 0.9187 & $\mathbf{0 . 0 3 1 4}$ & $* * *$ \\
\hline
\end{tabular}

Note. One-way permutational multivariate analysis of variance test, Permutation $N=9,999, P$ (same) $=.0002$.

Significantly $p$ values (<.05) are highlighted in bold.

Group labels follow Table 1.

shows that the Palaeolithic individuals are characterized by the most oblique wear, with significantly steeper wear facet planes than those of recent hunter-gatherers. Several authors have argued that the severity of dental wear in Plio-Pleistocene ancestors was not as exten-sive as in modern hunter-gatherer people where tooth wear resulted mainly from the excessive mixture of exogenous materials into their foods (Kaifu, 2000). In particular, Palaeolithic humans are characterized by steeper angles in Buccal and Lingual Phase I facets that are gener-ally formed during the shearing phase of the chewing cycle (Kay \& Hiiemae, 1974).

Because we did not find any major differences between groups that exploited different dietary sources, we need to take into account other possible factors, such as environmental abrasiveness and food processing methods, to explain why the Palaeolithic group is characterized by high wear angles. The analyses of cut marks, together with the discovery of fractured animal bones, suggest intentional butchering and marrow extraction (Mann, 2000). Moreover, although hearths and burned materials suggest controlled use of fire at many Neanderthal sites (Lev, Kislev, \& Bar-Yosef, 2005), we have little archaeological evidence of other food processing techniques with the exception of possible evidence of heating of grains (Henry, Brooks, \& Piperno, 2011; Mariotti Lippi, Foggi, Aranguren, Ronchitelli, \& Revedin,
2015). Moreover, although some scholars suggest that Neanderthals extracted lipid sources directly from bone gnawing and chewing (Costamagno, 2013; Marean, 2005), we do not find microtrauma patterns and large enamel chipping areas on Neanderthal postcanine teeth that indicate this behaviour (Fiorenza et al., 2015). We therefore assume that the diets of Palaeolithic humans, as highlighted by others (e.g., Kaifu, Kasai, Townsend, \& Richards, 2003), probably were not as abrasive as those of Arctic hunter-gatherers and Khoe-San people. This could be related to the food itself and/or to the use of less elab-orated food-processing methods (such as desiccation, frozening, and smoking) used by Palaeolithic humans, which probably introduced lower amounts of dust and grit in their daily diet.

Abrasion is the friction of exogenous materials forced over tooth surfaces (Kaidonis, 2008; Kaifu et al., 2003). The action of food on a tooth surface is not anatomically specific; the action generally occurs over the whole occlusal surface producing a wear area as opposed to a facet (Kaidonis, 2008). However, the abrasive action of food over the enamel surface tends to remodel the primary tooth morphology, reducing thus the slopes of the molar cusps, and therefore flattening the occlusal wear (Luke \& Lucas, 1983).

The different results between our and Smith's (1984) study could be related to two main aspects: first, lower accuracy in measuring the molar wear angles with 2D tools (which it becomes especially difficult in heavily worn teeth), second, and more important, the terms used by Smith (1984), such as "flat" versus "angled," do not refer to the same mechanisms analyzed in this study. Earlier works simply measured gross differences in cusp height between buccal and lingual sides of the tooth as wear progressed to extreme, leaving a flattened tooth ver-sus a tooth with markedly heavy wear on one side. The OFA approach takes place at a much finer scale than 1980s "wear plane angle" stud-ies. Here, we only considered wear facet angles associated to the different phases of the masticatory cycle, independent from cusp morphology (e.g., Fiorenza, Benazzi, \& Kullmer, 2011). The information decoded in wear facet patterns is used to reconstruct major occlusal movements and sequential tooth-to-tooth contacts between upper and lower teeth, with the aim to show how contact areas are created and what movements are responsible for their formation (e.g., Kullmer et al., 2009).

\subsection{Dental macrowear in modern hunter-gatherers and proto-farmers}

Smith (1984) hypothesized that a diet composed largely of ground grains and cooked (and therefore softer) foods had the effect of reduc-ing food toughness (and hence leading to a reduction in perpendicular 
masticatory forces) resulting in a more oblique wear. Furthermore, Smith (1984) noted that different hunter-gatherer groups exhibited similar wear plane angles despite regional differences in diet. Our results partially confirm these two main findings. For example, if we look at the wear facet plane angles on a broader scale, we can differen-tiate between traditional hunter-gatherers (flat macrowear patterns) and proto-farmers such as the Natufians (more oblique wear). Although the Natufians are considered hunter-gatherers who lived during the transitional period just before the Neolithic Revolution, they were living in sedentary communities, cultivating wild plants, harvest-ing cereals, and processing them with grinding stones. For this reason, they have been considered farmers by many, with a lifestyle and tech-nology significantly different from those of traditional hunter-gatherer communities (Bar-Yosef, 2011; Bowles \& Choi, 2013). The steeper wear facet planes of proto-farmer lingual facets found in this study could indicate either a dietary difference with the modern hunter-gath-erer group or the consumption of more refined and softened foods.

The overall flat occlusal wear found in our MHG sample may have been caused by both intrinsic and extrinsic abrasiveness of their diet and by different cultural habits. Inuit and Vancouver Islanders con-sumed foods low in abrasiveness such as meat and fish (Ames \& Maschner, 1999; Baarrengaard, 1949; El Zaatari, 2008, 2010; Gotfredsen, 1997; Pedersen, 1947; Pedersen \& Scott, 1951). Meat itself, for instance, is not hard enough to scratch the enamel surface. Differences in microwear signatures among populations with a diet mostly consisting of meat have been generally attributed to variations in the amount of physical properties of exogenous abrasive particles, either present in the environment or introduced through food process-ing methods (El Zaatari, 2008; Kaidonis, 2008; Kaifu et al., 2003; Lucas et al., 2013; Luke \& Lucas, 1983; Organ, Teaford, \& Larsen, 2005; Teaford, Larsen, Pastor, \& Noble, 2001).

Inuit populations, for example, consumed high amounts of raw, dried, or frozen meat (Baarrengaard, 1949; Gotfredsen, 1997; Pedersen, 1947; Pedersen \& Scott, 1951). They also extracted marrow from crushing and chewing bones, a behaviour that put an enormous pressure on their teeth causing the formation of large enamel chipping areas (Turner II \& Cadien, 1969). Raw meat requires a long chewing time before it can be reduced to smaller pieces and swallowed, overall increasing the masticatory effort (Zink \& Lieberman, 2016; Zink, Lieberman, \& Lucas, 2014). Because attritional wear facets are mostly produced by tooth-to-tooth contacts, prolonged mastication can increase the number of repetitive contact loadings between upper and lower teeth, and thus, leading to an increased degree of wear and flattening of the occlusal surface (Borrero-Lopez, Pajares, Constantino, \& Lawn, 2014; Brace, 1977; Hylander, 1976; Lucas, 2004). This effect can be easily seen in modern human societies with the intake of soft foods in their diets, which significantly decrease the amount of time we chew. This is also reflected in our dentition, which is characterized by very slightly worn teeth if compared with hunter-gatherer populations that relied on diets that required exten-sive and powerful chewing (Dahl, Carlsson, \& Ekfeldt, 1993; Kaifu, 2000; Kaifu et al., 2003; Kullmer et al., 2009; Scott \& Turner II, 1988).

Similarly, Vancouver Islanders used to store large quantities of salmon in drying and smoking houses (Ames \& Maschner, 1999). Meat (or fish) desiccation induces a significant change in its physical properties causing an increase in toughness (Molnar, 1972). All these daily activities and food preparation methods employed by Inuit and Vancouver Islander populations, besides changing food physical properties and increasing their toughness, seem to have also favoured the occasional ingestion of exogenous materials such as dust and grit, which may have significantly increased the amount of enamel loss.

Although the Khoe-San did not dry their meat nor cook it in earth ovens (Lee, 1973, 1978; Lee \& DeVore, 1976; O'Dea et al., 1991; Sahlins, 1968; Silberbauer, 1981), their teeth still exhibit an extremely flat wear. Moreover, because they mostly inhabited an arid and sandy environment, such as the Kalahari region, we should also take into account the importance of grit and dust present in the environment. As highlighted by Kaidonis (2008), in hunter-gatherers from desert, the environment abrasion represents the most prevalent mechanism of wear. Thus, we can hypothesize that the flat wear patterns found in our Khoe-San sample could be due to the combination of the masti-cation of hard food items and foreign small particles unintentionally introduced in their mouth. Finally, Natufian people did not process their foods as Arctic hunter-gatherers did, and besides the introduction of grinding stones, their diet was significantly less abrasive than the diets consumed by the Inuit, Vancouver Islanders, and Khoe-San (Bar-Yosef, 2011; Bowles \& Choi, 2013).

We need to stress here that, due to limitations in the method, we could only use slightly worn teeth, preventing thus the analysis of larger comparative samples. Because hunter-gatherers and prehistoric humans were characterized by extensive tooth wear, our approach can be limited to relatively young individuals and thus does not reflect the overall pattern found in one specific population. Moreover, it would be interesting to investigate if there were any signals in dental wear pat-tern that could show a difference or a division of labour by age and sex. Thus, future studies will necessitate the use of a larger sample size for better understanding the relationships between dental macrowear, chewing behaviour, culture, and diet. Moreover, they should also focus on potentially different patterns based on the stone-tool technology used and other functional aspects. For example, El Zaatari and Hublin (2014) found that changes in microwear texture signals of modern human teeth can be associated with major technological complexes of the Upper Paleolithic. More specifically, the analysis of the microwear texture showed significant differences between individuals from Aurignacian and Gravettian contexts relative to those of Magdalenian culture, with the latter characterized by a more abrasive diet (El Zaatari \& Hublin, 2014). Finally, it would be interesting as well, to investigate how wear plane angles vary at different wear stages.

\section{ACKNOWLEDGEMENTS}

The authors gratefully thank the curators and institutions which allowed us to study comparative and fossil specimens: Almut Hoffmann (Museum für Vor- und Frühgeschichte, Berlin, Germany), Maria Teschler Nicola (Naturhistorisches Museum Wien, Vienna, Austria), Marta Dočkalová (Moravské Zemské Muzeum, Anthropos Institute, Brno, Czech Republic), Bence Viola (Department of Anthropology, University of Vienna, Austria), Yoel Rak (Department of Anatomy and Anthropology, University of Tel Aviv, Israel), Fabio Parenti (Istituto Italiano di Paleontologia Umana, Rome, Italy), Chris 
Stringer and Rob Kruszynski (Natural History Museum of London, England), National Museum of Wales (Cardiff, Wales), Angiolo del Lucchese (Museo Preistorico dei Balzi Rossi, Ventimiglia, Italy), and Erik Trinkaus (Department of Physical Anthropology at Washington University in Saint Louis, USA). We also would like to thank Christine Hemm for her help in surface scanning the original and cast dentition included in this sample and Iva Nikolic for copy-editing our manuscript. This study was supported by the Faculty of Medicine, Nursing and Health Sciences at Monash University through the Strategic Grant Scheme 2016 (Grant SGS16-0344)

\section{ORCID}

Luca Fiorenza http://orcid.org/0000-0001-7110-3398

\section{REFERENCES}

Addy, M., \& Shellis, R. P. (2006). Interaction between attrition, abrasion and erosion in tooth wear. Monographs in Oral Science, 20,17-31.

Ames, K. M., \& Maschner, D. G. (1999). Peoples of the Northwest Coast Their archaeology and prehistory. London: Thames and Hudson.

Baarrengaard, D. D. S. (1949). Dental conditions and nutrition among natives in Greenland. Oral Surgery, Oral Medicine, Oral Pathology, 2, 995-1007.

Bar-Yosef, O. (2011). The origins of agriculture: New data, new ideas. Current Anthropology, 52, S175-S193.

Borrero-Lopez, O., Pajares, A., Constantino, P. J., \& Lawn, B. R. (2014) A model for predicting wear rates in tooth enamel. Journal of the Mechanical Behavior of Biomedical Materials, 37, 226-234.

Bowles, S., \& Choi, J. K. (2013). Coevolution of farming and private prop-erty during the early Holocene. Proceedings of the National Academy of Sciences of the United States of America, 110, 8830-8835.

Brace, C. L. (1977). Occlusion to the anthropological eye. In J. A. McNamara (Ed.), The biology of occlusal development ()Monograph No. 7, Craniofacial Growth Series (pp. 179-209). Ann Arbor: Center for Human Growth and Development, the University of Michigan.

Caspari, R., \& Lee, S-H. (2004). Older age becomes common late in human evolution. Proceedings of the National Academy of Sciences of the United States of America, 101, 10895-10900.

Costamagno, S. (2013). Bone grease rendering in Mousterian contexts: the case of Noisetier Cave (Fréchet-Aure, Hautes-Pyrénées, France). In J. L. Clark, \& J. D. Speth (Eds.), Zooarchaeology and modern human origins: Human hunting behavior during the Later Pleistocene (pp. 209-225). New York: Springer.

Dahl, B., Carlsson, G. E., \& Ekfeldt, A. (1993). Occlusal wear of teeth and restorative materials: A review of classification, etiology, mechanisms of wear, and some aspects of restorative procedures. Acta Odontologica Scandinavica, 51, 299-311.

El Zaatari, S. (2008). Occlusal molar microwear and the diets of the Ipiutak and Tigara populations (Point Hope) with comparisons to the Aleut and Arikara. Journal of Archaeological Science, 35, 2517-2522.

El Zaatari, S. (2010). Occlusal microwear texture analysis and the diets of historical/prehistoric hunter-gatherers. International Journal of Osteoarchaeology, 20, 67-87.

El Zaatari, S., Grine, F. E., Ungar, P. S., \& Hublin, J. J. (2011). Ecogeographis variation in Neandertal dietary habits: Evidence from occlusal molar microwear texture analysis. Journal of Human Evolution, 61, 411-424.

El Zaatari, S., \& Hublin, J. J. (2014). Diet of upper Paleolithic modern humans: Evidence from microwear texture analysis. American Journal of Physical Anthropology, 153, 570-581.

Every, R. G. (1972). A new terminology for mammalian teeth: Founded on the phenomenon of thegosis. Parts 1 \& 2. Pegasus: Christchurch.
Fiorenza, L. (2009). Occlusal wear pattern analysis of functional morphology in Neanderthals and early Homo sapiens dentition (Ph.D. dissertation). Frank-furt: Johann Wolfgang Goethe University.

Fiorenza, L. (2015). Reconstructing diet and behaviour of Neanderthals from Central Italy through dental macrowear analysis. Journal of Anthro-pological Sciences, 93, 1-15.

Fiorenza L, Benazzi S, Henry A, Salazar-García DC, Blasco R, Picin A, Wroe S, Kullmer O. 2015. To meat or not to meat? New perspectives on Neanderthal ecology. Yearbook of Physical Anthropology 156, Supple-ment S59: 43-71.

Fiorenza, L., Benazzi, S., \& Kullmer, O. (2009). Morphology, wear and 3D digital surface models: Materials and techniques to create high-resolu-tion replicas of teeth. Journal of Anthropological Sciences, 87, 211-218.

Fiorenza, L., Benazzi, S., \& Kullmer, O. (2011). Para-masticatory wear facets and their functional significance in hunter-gatherer maxillary molars. Journal of Archaeological Science, 38, 2182-2189.

Fiorenza, L., Benazzi, S., Tausch, J., Kullmer, O., Bromage, T. G., \& Schrenk, F. (2011). Molar macrowear reveals Neanderthal eco-geographical die-tary variation. PLoS One, 6, e14769.

Fiorenza, L., Benazzi, S., Tausch, J., Kullmer, O., \& Schrenk, F. (2010). Brief communication: Identification reassessment of the isolated tooth Krapina D58 through occlusal fingerprint analysis. American Journal of Physical Anthropology, 143, 306-312.

Fiorenza, L., Benazzi, S., Viola, B., Kullmer, O., \& Schrenk, F. (2011). Rela-tionship between cusp size and occlusal wear pattern in Neanderthal and Homo sapiens first maxillary molars. The Anatomical Record, 294, 453-461.

Fiorenza, L., \& Kullmer, O. (2013). Dental wear and cultural behaviour in middle Paleolithic humans from the near east. American Journal of Physical Anthropology, 152, 107-117.

Fiorenza, L., \& Kullmer, O. (2015). Dental wear patterns in early modern humans from Skhul and Qafzeh: A response to Sarig and Tillier. Homo, 66, 414-419.

Fiorenza, L., \& Kullmer, O. (2016). Occlusion in an adult male gorilla with a supernumerary maxillary premolar. International Journal of Primatology, 37, 762-777.

Gotfredsen, A. B. (1997). Sea bird exploitation on coastal Inuit sites, west and southeast Greenland. International Journal of Osteoarchaeology, 7, 271-286.

Grine, F. E. (1986). Dental evidence for dietary differences in Australopithecus and Paranthropus: A quantitative analysis of permanent molar microwear. J. Hum. Evol, 15, 783-822.

Hammer, Ø., Harper, D. A. T., \& Ryan, P. D. (2001). PAST: Paleontological Statistics software package for education and data analysis. Palaentologia Electronica, 4, 9.

Harvati, K., Darlas, A., Bailey, S. E., Rein, T. R., el Zaatari, S., Fiorenza, L., ... Psathi, E. (2013). New Neanderthal remains from Mani peninsula, S. Greece: The Kalamakia Middle Palaeolithic cave site. J. Hum. Evol, 64, 486-499.

Henry, A. G., Brooks, A. S., \& Piperno, D. R. (2011). Microfossils in calculus demonstrate consumption of plants and cooked foods in Neanderthal diets (Shanidar III, Iraq; Spy I and II, Belgium). Proceedings of the National Academy of Sciences of the United States of America, 108, 486-491.

Hinton, R. (1982). Differences in interproximal and occlusal tooth wear among prehistoric Tennessee Indians: Implications for masticatory func-tion. American Journal of Physical Anthropology, 57, 103-115.

Hylander, W. L. (1976). Morphological changes in human teeth and jaws in a high-attrition environment. In A. A. Dahlberg, \& T. M. Graber (Eds.), Orofacial growth and development (pp. 301-330). The Hague: Mouton.

Janis, C. M. (1990). The correlation between diet and dental wear in herbivorous mammals, and its relationship to the determination of diets of extinct species. In A. J. Boucot (Ed.), Evolutionary Paleobiology of Behav-ior and Coevolution (pp. 241-259). Amsterdam: Elsevier Science.

Kaidonis, J. A. (2008). Tooth wear: The view of the anthropologist. Clin. Oral. Invest. Supp. 1, 12, 21-26. 
Kaidonis, J. A., Townsend, G. C., \& Richards, L. C. (1993). Nature and fre-quency of dental wear facets in an Australian Aboriginal population. Journal of Oral Rehabilitation, 20, 333-340.

Kaifu, Y. (2000). Was extensive tooth wear normal in our ancestors?: A preliminary examination in the genus Homo. Anthropological Science, 108, 471-385.

Kaifu, Y., Kasai, K., Townsend, G. C., \& Richards, L. C. (2003). Tooth wear and the "design" of the human dentition: A perspective from evolution-ary medicine. Yearbook of Physical Anthropology, 46, 47-61.

Kay, R. F. (1977). The evolution of molar occlusion in the Cercopithecidae and early catarrhines. American Journal of Physical Anthropology, 46, 327-352.

Kay, R. F., \& Hiiemae, K. M. (1974). Jaw movement and tooth use in recent and fossil primates. American Journal of Physical Anthropology, 40, 227-256.

Kullmer, O., Benazzi, S., Fiorenza, L., Schulz, D., Bacso, S., \& Winzen, O. (2009). Technical note: Occlusal fingerprint analysis: Quantification of tooth wear pattern. American Journal of Physical Anthropology, 139, 600-605.

Le Luyer, M., Rottier, S., \& Bayle, P. (2014). Brief communication: Compar-ative patterns of enamel thickness topography and oblique molar wear in two Early Neolithic and medieval population samples. American Journal of Physical Anthropology, 155, 162-172.

Lee, R. B. (1973). Mongongo: Ethnography of a major wild food resource. Ecology of Food and Nutrition, 2, 307-321.

Lee, R. B. (1978). Ecology of a contemporary san people. In P. Tobias (Ed.), The bushmen: san hunters and herders of Southern Africa (pp. 98-114). Cape town: Human and Rousseau.

Lee, R. B., \& DeVore, I. (1976). Kalahari Hunter-Gatherers: Studies of the! Kung San and their neighbors. Cambridge: Harvard University Press.

Lev, E., Kislev, M. E., \& Bar-Yosef, O. (2005). Mousterian vegetal food in Kebara Cave, Mt. Carmel. Journal of Archaeological Science, 32, 475-484.

Lucas, P. W. (2004). Dental functional morphology: How teeth work. Cambridge, UK: Cambridge University Press.

Lucas, P. W., Omar, R., Al-Fadhalah, K., Almusallam, A. S., Henry, A. G., Michael, S., ... Atkins, A. G. (2013). Mechanisms and causes of wear in tooth enamel: Implications for hominin diets. J. R. Soc. Interface, 10, 20120923.

Luke, D. A., \& Lucas, P. W. (1983). The significance of cusps. Journal of Oral Rehabilitation, 10, 197-206.

Maier, W., \& Schneck, G. (1981). Konstruktionsmorphologische Untersuchungen am Gebiß der hominoiden Primaten. Z. Morph. Anthrop., 72, 127-169.

Mann, N. (2000). Dietary lean red meat and human evolution. European Journal of Nutrition, 39, 71-79.

Marean, C. W. (2005). From the tropics to the colder climates: Contrasting faunal exploitation adaptations of modern humans and Neanderthals. In F. D'Errico, \& L. Backwell (Eds.), From tools to symbols: From early hom-inids to humans (pp. 333-371). Johannesburg: Witwatersrand University Press.

Mariotti Lippi, M., Foggi, B., Aranguren, B., Ronchitelli, A., \& Revedin, A. (2015). Multistep food plant processing at Grotta Paglicci (Southern Italy) around 32,600 cal B.P. Proceedings of the National Academy of Sciences of the United States of America, 112, 12075-12080.

Molnar, S. (1971). Human tooth wear, tooth function and cultural variability. American Journal of Physical Anthropology, 34, 175-189.

Molnar, S. (1972). Tooth wear and culture: A survey of tooth functions among some prehistoric populations. Current Anthropology, 13, 511526
O'Dea, K., Jewell, P. A., Whiten, A., Altmann, S. A., Strickland, S. S., \& Oftedal, O. T. (1991). Traditional diet and food preferences of Austra-lian Aboriginal hunter-gatherers. Phyl. Trans. Biol. Sci., 334, 233-241.

Organ, J. M., Teaford, M. F., \& Larsen, C. S. (2005). Dietary inferences from dental occlusal microwear at Mission San Luis de Apalachee. American Journal of Physical Anthropology, 128, 801-811.

Oxilia, G., Peresan, M., Romandini, M., Matteucci, C., Spiteri, C., Henry, A., ... Benazzi, S. (2015). Earliest evidence of dental treatment in the Late Upper Paleolithic. Scientific Reports, 5, 12150.

Pedersen, P. O. (1947). Dental investigations of Greenland Eskimos. Proceedings of the Royal Society of Medicine, 40, 726-732.

Pedersen, P. O., \& Scott, D. B. (1951). Replica studies of the surfaces of teeth from Alaskan Eskimos. West Greenland natives and American whites. Acta Odontologica Scandinavica, 9, 262-292.

Sahlins MD. 1968. Notes on the original affluent society. Man the hunter, RB Lee, I. De Vore (eds.). Aldine publishing company: Chicago, 85-89.

Scott, R. G., \& Turner, G. II (1988). Dental anthropology. Annual Review of Anthropology, 17, 99-126.

Silberbauer, G. (1981). Hunter and habitat in the Central Kalahari Desert. Cambridge: Cambridge University Press.

Smith, B. H. (1984). Patterns of molar wear in hunter-gatherers and agriculturists. American Journal of Physical Anthropology, 63, 39-56.

Teaford, M. F., Larsen, C. S., Pastor, R. F., \& Noble, V. E. (2001). Pits and scratches. Microscopic evidence of tooth use and masticatory behav-iour in La Florida. In C. S. Larsen (Ed.), Bioarchaeology of La Florida: Human biology in the northern frontier New Spain (Vol. 82) (pp. -112). Gainesville: University Press of Florida.

Turner, C. G. II, \& Cadien, J. D. (1969). Dental chipping in Aleuts, Eskimos and Indians. American Journal of Physical Anthropology, 31, 303-310.

Ulhaas, L., Kullmer, O., \& Schrenk, F. (2007). Tooth wear diversity in early hominid molars: A case study. In S. E. Bailey, \& J. J. Hublin (Eds.), Dental perspectives on human evolution: State of the art research in dental paleo-anthropology (pp. 369-390). The Netherlands: Springer.

Watson, J. T. (2008). Changes in food processing and occlusal dental wear during the early agricultural period in northwest Mexico. American Jour-nal of Physical Anthropology, 135, 92-99.

Zink, K. D., \& Lieberman, D. E. (2016). Impact of meat and Lower Palaeolithic food processing techniques on chewing in humans. Nature, 531, 500-505.

Zink, K. D., Lieberman, D. E., \& Lucas, P. W. (2014). Food material proper-ties and early hominin processing techniques. Journal of Human Evolution, 77, 155-166. 